\title{
Article \\ Electronic and Structural Properties of the Double Cubane Iron-Sulfur Cluster
}

\author{
Nadia Elghobashi-Meinhardt *, Daria Tombolelli and Maria Andrea Mroginski
}

Citation: Elghobashi-Meinhardt, N.; Tombolelli, D.; Mroginski, M.A.

Electronic and Structural Properties of the Double Cubane Iron-Sulfur Cluster. Catalysts 2021, 11, 245. https://doi.org/10.3390/ catal11020245

Academic Editor:

Maria-Eirini Pandelia

Received: 13 January 2021

Accepted: 5 February 2021

Published: 12 February 2021

Publisher's Note: MDPI stays neutral with regard to jurisdictional claims in published maps and institutional affiliations.

Copyright: (c) 2021 by the authors. Licensee MDPI, Basel, Switzerland. This article is an open access article distributed under the terms and conditions of the Creative Commons Attribution (CC BY) license (https:// creativecommons.org/licenses/by/ $4.0 /)$.
Department of Chemistry, Technische Universität Berlin, Straße-des-17. Juni 135, 10623 Berlin, Germany; tombolelli.daria@gmail.com (D.T.); andrea.mroginski@tu-berlin.de (M.A.M.)

* Correspondence: n.elghobashi-meinhardt@campus.tu-berlin.de; Tel.: +49-30-3417-9386

Abstract: The double-cubane cluster (DCC) refers to an $\left[\mathrm{Fe}_{8} \mathrm{~S}_{9}\right]$ iron-sulfur complex that is otherwise only known to exist in nitrogenases. Containing a bridging $\mu_{2}-S$ ligand, the DCC in the DCCcontaining protein (DCCP) is covalently linked to the protein scaffold via six coordinating cysteine residues. In this study, the nature of spin coupling and the effect of spin states on the cluster's geometry are investigated computationally. Using density functional theory (DFT) and a broken symmetry (BS) approach to study the electronic ground state of the system, we computed the exchange interaction between the spin-coupled spins of the four FeFe dimers contained in the DCC. This treatment yields results that are in excellent agreement with both computed and experimentally determined exchange parameters for analogously coupled di-iron complexes. Hybrid quantum mechanical (QM)/molecular mechanical (MM) geometry optimizations show that cubane cluster A closest to charged amino acid side chains (Arg312, Glu140, Lys146) is less compact than cluster $B$, indicating that electrons of the same spin in a charged environment seek maximum separation. Overall, this study provides the community with a fundamental reference for subsequent studies of DCCP, as well as for investigations of other $\left[\mathrm{Fe}_{8} \mathrm{~S}_{9}\right]$-containing enzymes.

Keywords: [Fe8-S9] iron-sulfur cluster; double-cubane cluster (DCC); broken symmetry (BS); density function theory (DFT); quantum mechanics (QM)/molecular mechanics (MM); geometry optimizations; exchange parameters

\section{Introduction}

Enzymes catalyzing ATP-driven redox reactions usually consist of two components. The first component is an ATPase, which contains an FeS cluster that provides the electron that is to be transferred. The second component is another metalloenzyme, the electron acceptor, which is ready to accept a highly energetic electron since it contains one or more metal centers that cannot easily be reduced at physiological reduction potentials [1]. Recently, the two components of a system of this type were characterized and the electronaccepting component was found to be a novel double-cubane [ $\left.\mathrm{Fe}_{8} \mathrm{~S}_{9}\right]$-cluster (DCC) [1] (Figure 1). This cluster catalyzes reductive reactions otherwise associated only with the complex iron-sulfur clusters of nitrogenases [2]. Although double-cubane $\left[\mathrm{Fe}_{8} \mathrm{~S}_{9}\right]$-clusters have been reported only once for a biological system, they have already been synthesized and studied [3-6].

Stack et al. reported the first example of a sulfide-bridged double-cubane cluster in 1989, hypothesizing that such a Fe-S complex may exist in proteins, as it is constructed of discrete cubane units [3,7]. Several variants of the inorganic DCC with different ligands have been studied, showing the lability of the bridging $\mu_{2}-S[3,4,7,8]$ and very weak magnetic coupling between the two subclusters. However, the electron transfer is strongly coupled and two distinct redox reactions occur [3]. If such a FeS cluster is embedded in a so-called "double-cubane cluster" protein (DCCP), diverse environments of the two subclusters may lead to differing reduction potentials [1]. 


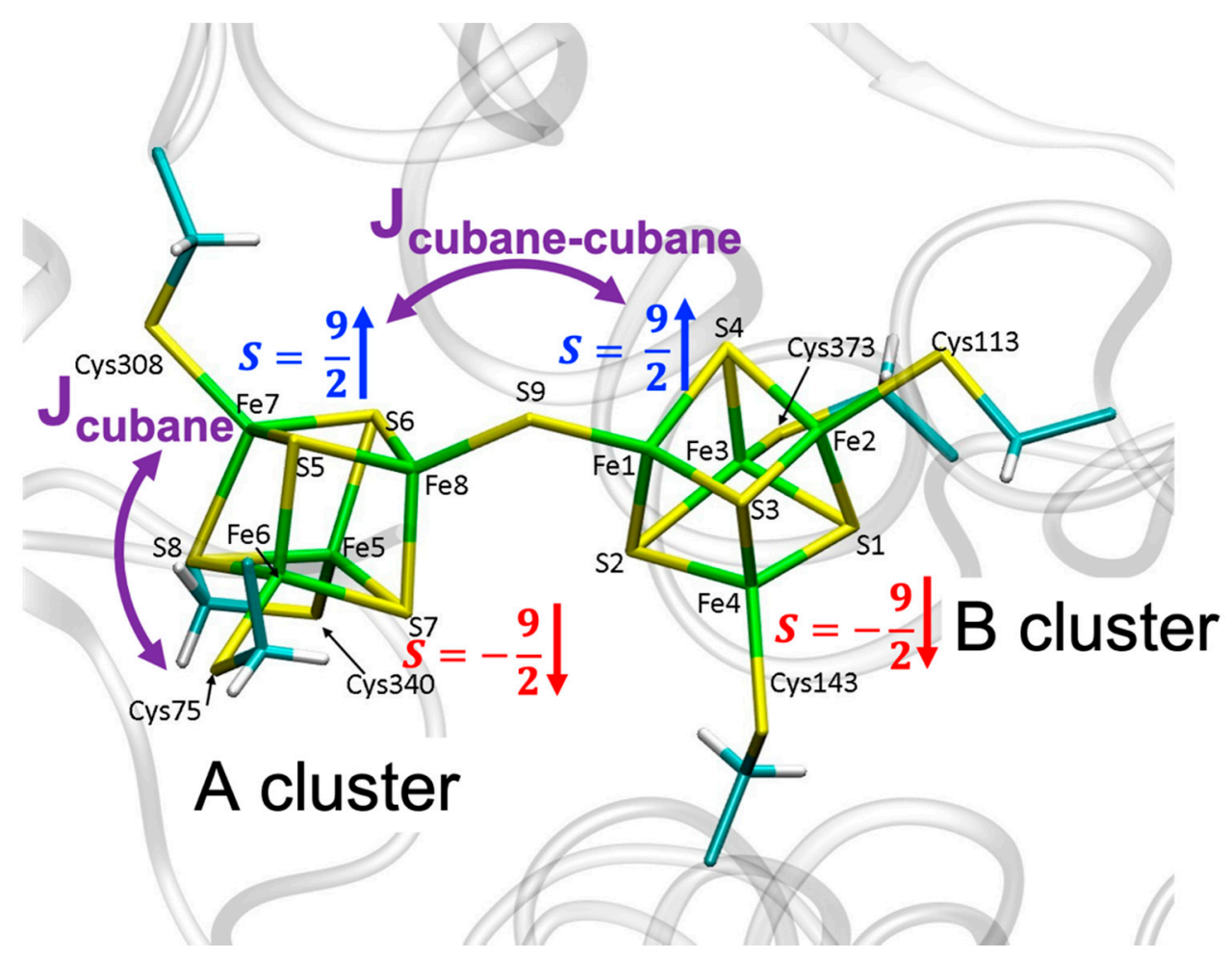

Figure 1. The double-cubane cluster (DCC) of the double-cubane cluster protein (DCCP), from the structure file 6ENO.pdb $(1.63 \AA$ ) [1], shows the A-cluster coordinated to sidechains of Cys75, Cys308, and Cys340 and the B-cluster coordinated to sidechains of Cys113, Cys143, and Cys373. A-cluster is linked to B-cluster via the $\mu_{2}-\mathrm{S}$ ligand (S9). Each cubane cluster contains an exchange interaction between the mixed-valence Fe dimers within the cubane (J $\left.\mathrm{J}_{\text {cubane }}\right)$ (dimer high spin "up" shown in blue; dimer high spin "down" shown in red). An exchange energy also arises from two mixed-valence Fe dimers interacting via the $\mu_{2}-\mathrm{S}$ ligand (J cubane-cubane).

Notably, the corresponding cluster in Carboxydothermus hydrogenoformans, $\mathrm{DCCP}_{\mathrm{Ch}}$, exhibits similarities regarding structure and reactivity with the FeMoCo iron-molybdenum cofactor of nitrogenases [9]. Both enzymes contain two Rossmann-fold domains with similar orientation, thereby providing the six coordinating Cys residues [1]. Furthermore, both enzymes are capable of an ATP-dependent two electron reduction of acetylene and hydrazine [1]. Such a two-electron reduction, so far only observed in nitrogenases, is believed to start with acetylene binding to the metal cluster and transiently acting as a bridging ligand between two adjacent Fe-ions, which are $3.9 \AA$ apart from each other [10]. A similar distance (3.74 $\AA$ ) exists between $\mathrm{Fe} 1$ and $\mathrm{Fe} 8$ in $\mathrm{DCCP}_{\mathrm{Ch}}$, bridged by $\mu_{2}-\mathrm{S}$, and facing the potential substrate channel [1]. Hence, this analogy with nitrogenases led to speculation that during catalysis the bridging sulfide site may dissociate leaving space for acetylene to bind $\mathrm{Fe} 1$ and Fe8, activating catalysis [1]. Further evidence that DCCP may function analogously to nitrogenase comes from inhibition experiments. As in nitrogenase, $\mathrm{DCCP}_{\mathrm{Ch}}$ reduction of acetylene is reversibly inhibited by $\mathrm{CO}$; this inhibition is speculated to occur when $\mathrm{CO}$ replaces the labile ligand [1].

For metal clusters, in which multiple iron centers are joined by sulfur atoms with a variety of geometric arrangements, an accurate description of the electronic structure is required. The electronic state of the transition metal cluster may have a fundamental influence, not only on the geometry, but also on the spectroscopic properties of the cluster. Different well-established experimental techniques exist for probing the various states of the catalytic cycle, as well as the physicochemical properties of FeS clusters. Among those, electron paramagnetic resonance (EPR) [10-12], infrared IR [13-16], resonance Raman (RR) [13-17], and Mössbauer [18] spectroscopy are powerful complementary techniques allowing the description of structural and electronic properties of the metal clusters [19]. EPR, based on microwave radiation, is well-suited to studying systems containing unpaired 
electrons, common for high-spin Fe centers [20]. Mössbauer spectroscopy relies on the resonant and recoil-free emission and absorption of gamma radiation by a chemical species. The gamma rays are accelerated through a range of velocities and the detected intensity is plotted as a function of the source velocity. When gamma rays are absorbed by the sample, a drop in measured intensity occurs. The Mössbauer spectrum thus reveals information about the chemical environment of the absorbing nuclei $\left({ }^{57} \mathrm{Fe}\right)[18]$.

The most common theoretical method for describing metal clusters containing multiple iron centers is the broken symmetry (BS) approach [21-23]. The need for a BS treatment is that electronic exchange interactions among multiple Fe centers are difficult to treat with density functional theory (DFT), which is limited to single-determinant wave functions. The BS methodology attempts to capture the multiple-determinant ground-state wave function by using a weighted average of pure spin states arising from a specific distribution of localized electrons. Alternatively, more recently, a type of multireference configuration interaction (CI) approach has been applied to treat coupled Fe spin states, relying on a finite complete active space (CAS) of interacting iron and sulfur orbitals $[24,25]$. To treat the $\left[\mathrm{Fe}_{2} \mathrm{~S}_{2}\right]^{2+}$ cluster correctly, the minimum CAS of all $3 \mathrm{~d}$ orbitals of iron atoms and 3p orbitals of the bridging sulfur atoms would lead to 16 orbitals containing 22 electrons. Sharma et al. applied a theory termed density matrix renormalization group (DMRG) to treat minimal and extended CAS of $\left[\mathrm{Fe}_{2} \mathrm{~S}_{2}\right]$ and $\left[\mathrm{Fe}_{4} \mathrm{~S}_{4}\right]$ clusters [26]. Others have since applied similar methodology to study excited states of iron-sulfur clusters in various oxidation and spin states $[27,28]$.

Here, we consider the double-cubane cluster of $\mathrm{DCCP}_{\mathrm{Ch}}$ (6ENO.pdb) [1] in a doubly oxidized state; i.e., each of the sub-clusters is in an oxidized state $\left[\mathrm{Fe}_{4} \mathrm{~S}_{4}\right]^{2+}$, consisting of two pairs of high-spin (HS) ferrous $\left(\mathrm{Fe}^{2+}, \mathrm{d} 6, S=2\right)$ and ferric $\left(\mathrm{Fe}^{3+}, \mathrm{d} 5, S=5 / 2\right)$ iron sites.

Therefore, for two Fe atoms, and analogously for four Fe atoms, the total $S=9 / 2$ dimer spins are antiferromagnetically coupled to produce the resulting $S=0\left(\mathrm{M}_{\mathrm{S}}=1\right)$ ground state (Figure 2).
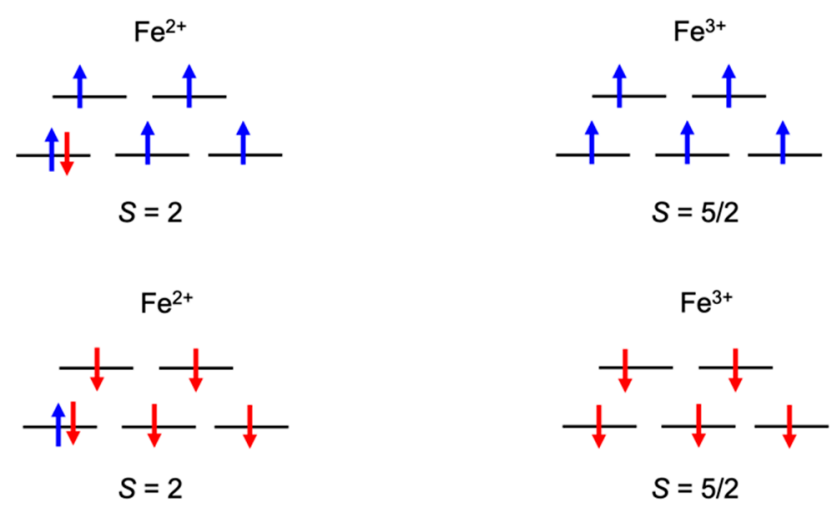

Figure 2. The scheme shows the antiferromagnetic coupling arrangement (total $S=0$ ) of four highspin iron centers consisting of two ferrous $\left(\mathrm{Fe}^{2+}, \mathrm{d} 6, S=4 / 2=2\right)$ iron centers and two ferric $\left(\mathrm{Fe}^{3+}, \mathrm{d} 5\right.$, $S=5 / 2$ ) iron centers.

Exchange interactions within the DCC cluster may be described by two predominant types of couplings: (1) within a single cubane, the two $\left[\mathrm{Fe}^{2+} \mathrm{Fe}^{3+}\right]$ dimers may couple $\left(\mathrm{J}_{\text {cubane }}\right)$; (2) between the two cubane clusters, two $\left[\mathrm{Fe}^{2+} \mathrm{Fe}^{3+}\right]$ dimers may couple via the bridging between $\mu_{2}-S$ atom (J $J_{\text {cubane-cubane }}$ ) (Figure 1$)$. This scheme is based on the assumption that the double-exchange interactions within the cubane are so strong that the mixed-valence $\left[\mathrm{Fe}^{2+} \mathrm{Fe}^{3+}\right]$ pair can be treated as a single spin, an assumption that is supported by spectroscopic studies [29,30]. A similar approach was successfully applied by Fiedler and Brunold in a "three-spin" model to study the exchange interactions between the cubane cluster $\left[\mathrm{Fe}_{4} \mathrm{~S}_{4}\right]$ and di-iron subunit $[2 \mathrm{Fe}]_{\mathrm{H}}$ within the Fe-only hydrogenase [31]. This model assumes that the interaction between distantly separated $\left[\mathrm{Fe}^{2+} \mathrm{Fe}^{3+}\right]$ dimers is negligible [31]. 
According to the Heisenberg-Dirac-Van Vleck (HDVV) Hamiltonian, the exchange coupling constant $\mathrm{J}$ can be related to the energy through the Hamiltonian $\mathrm{H}=\mathrm{J}\left(S_{\mathrm{A}} \cdot S_{\mathrm{B}}\right)$ where $S_{\mathrm{A}}$ and $S_{\mathrm{B}}$ are the spin operators for centers $\mathrm{A}$ and $\mathrm{B}$, respectively [21]. The Hamiltonian can be extended to account for double exchange via $\mathrm{H}=\mathrm{J}\left(S_{\mathrm{A}} \cdot S_{\mathrm{B}}\right) \pm \mathrm{B}\left(S_{\text {total }}+1 / 2\right)$, where $B$ is the resonance delocalization parameter [23]. If the mixed-valence $\left[\mathrm{Fe}^{2+} \mathrm{Fe}^{3+}\right]$ pair is treated as a single spin, the delocalization parameter B is not treated explicitly [31].

Given these assumptions, and following the "three-spin" model of Fielder and Brunold [31], a "four-spin" model accounting for the coupling of four spin systems can be constructed for the DCC system (Figure 3).

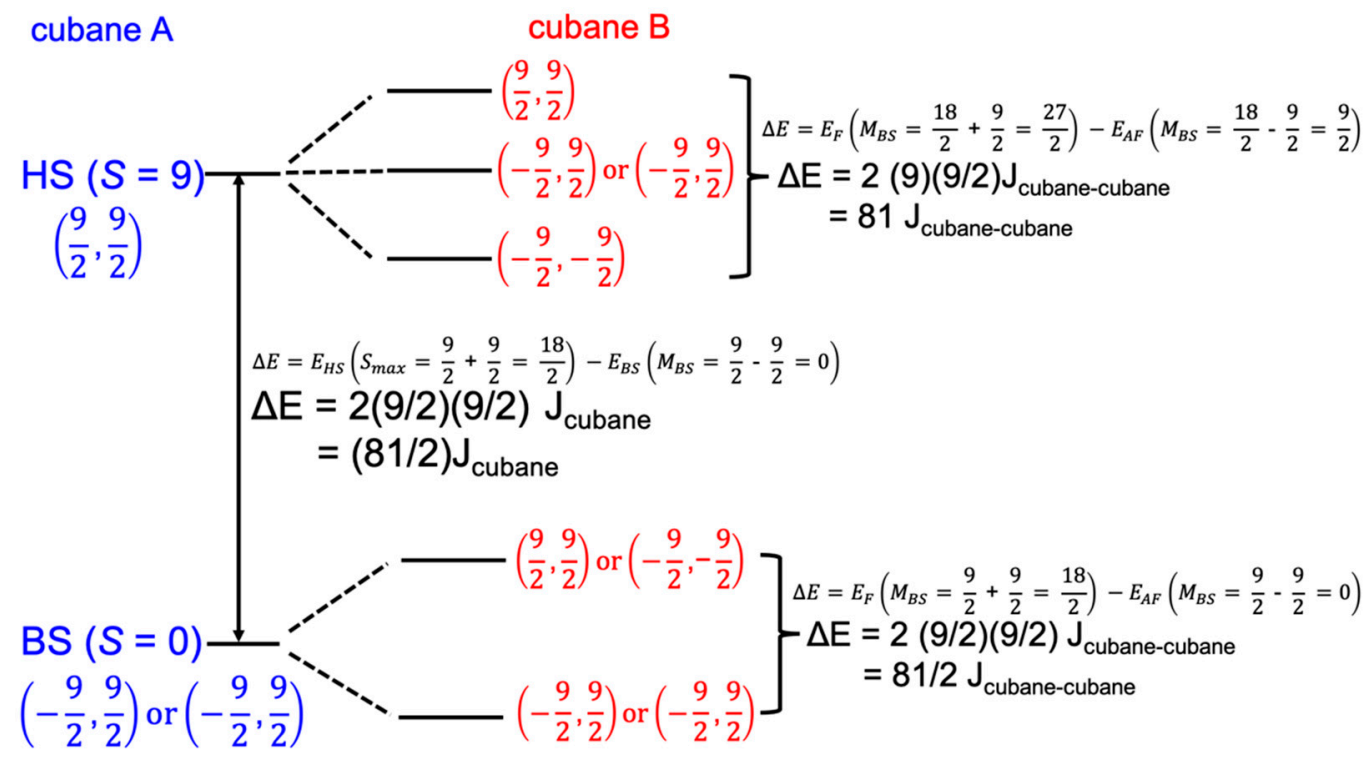

Figure 3. The scheme shows the possible spin coupling arrangements for the DCC, considered within a "four-spin model" (four $\left[\mathrm{Fe}^{2+} \mathrm{Fe}^{3+}\right]$ dimers). The blue labels correspond to the coupling of $\left[\mathrm{Fe}^{2+} \mathrm{Fe}^{3+}\right]$ pairs within the cubane $\left(\mathrm{J}_{\text {cubane }}\right)$, and the red labels show the possible couplings for $\left[\mathrm{Fe}^{2+} \mathrm{Fe}^{3+}\right]$ dimers via the bridging $\mu_{2}-\mathrm{S}$ atom $\left(\mathrm{J}_{\text {cubane-cubane }}\right)$. The "four-spin model" assumes the coupling between distant $\left[\mathrm{Fe}^{2+} \mathrm{Fe}^{3+}\right]$ pairs is negligible.

The spin coupling constant, J, which describes the exchange interaction between two spins $\left(S_{\mathrm{A}}, S_{\mathrm{B}}\right)$ can therefore be computed from the energies of the high spin (HS) and BS states via the following equation:

$$
\mathrm{E}_{\mathrm{HS}}\left(S_{\max }=S_{\mathrm{A}}+S_{\mathrm{B}}\right)-\mathrm{E}_{\mathrm{BS}}\left(\mathrm{M}_{\mathrm{BS}}=\left|S_{\mathrm{A}}-S_{\mathrm{B}}\right|\right)=2 \mathrm{~J} S_{\mathrm{A}} S_{\mathrm{B}}
$$

Here, only the HS state is an eigenfunction of $S^{2}$ and the BS state, as it is not a pure spin state, is an eigenfunction of $\mathrm{M}_{\mathrm{S}}$.

Multiple BS states can be conceived for eight Fe ions. Here, in addition to two high spin states ( $\alpha \alpha \alpha \alpha-\alpha \alpha \alpha \alpha$ and $\alpha \alpha \alpha \alpha-\beta \beta \beta \beta), 22$ BS states were modeled in order to determine the electronic ground state of $\mathrm{DCCP}_{\mathrm{Ch}}$ and the magnitude of $\mathrm{J}_{\text {cubane }}$ and $\mathrm{J}_{\text {cubane-cubane }}$ (Figure 4). 


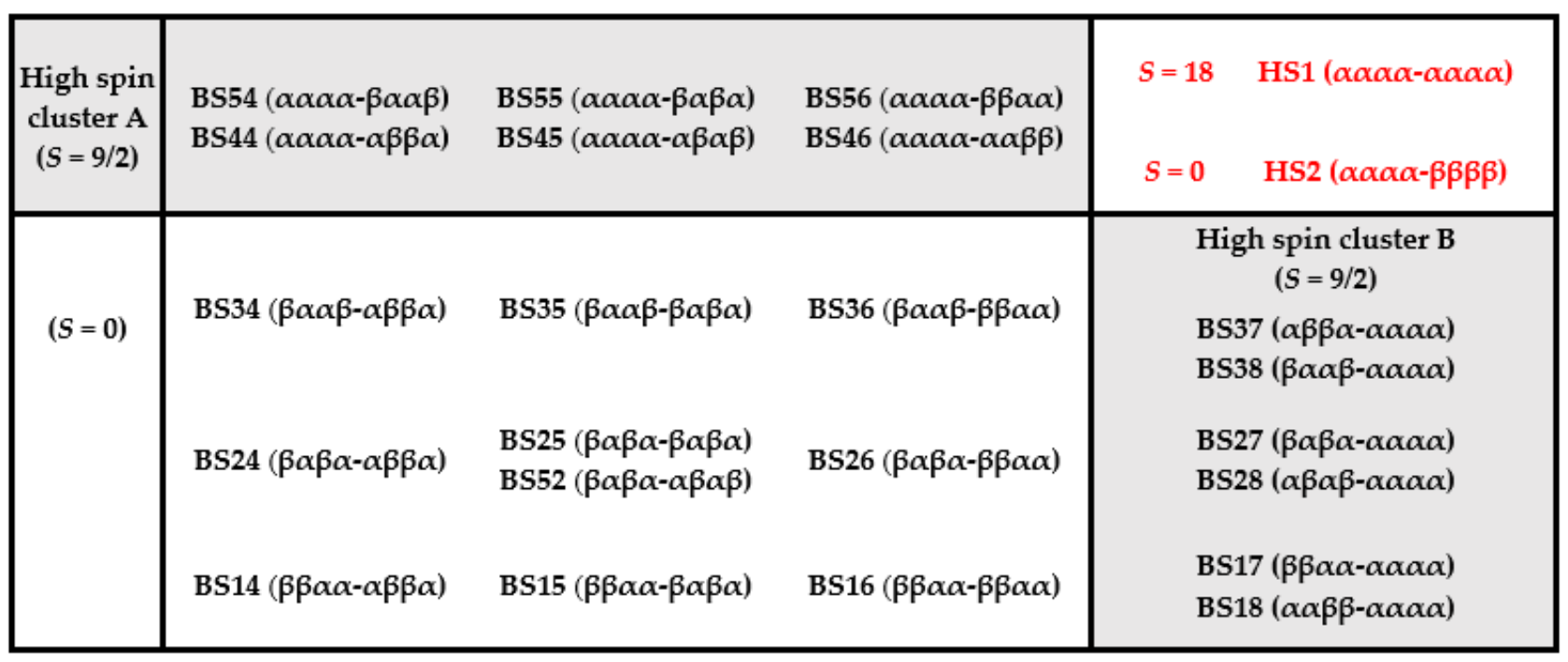

Figure 4. Broken symmetry (BS) configurations. The 24 spin states studied are listed with the notation xxxx-xxxx corresponding to the spin on each Fe atom (1234-5678), where Fe1 and Fe8 are the two bridging Fe atoms (Figure 1). The white central panel lists ten low spin BS states $(S=0)$, while the grey shaded areas contain BS states in which one cluster is high spin and the other cluster is low spin $(S=9 / 2)$, either $\alpha \alpha$ or $\alpha \beta$ across the bridging $\mu_{2}-S$. The two high spin states $(S=18$ and $S=0$ ) are listed in red in the top right corner.

These 24 states are constructed to probe the wavefunction stability as a function of spin distribution within the DCC. Please note here that our spin notation ( $x x x x-x x x x)$ corresponds to Fe atoms (1234-5678), where Fe1 and Fe8 are the two bridging Fe atoms (Figure 1). Therefore, states with $\alpha x x x-x x x \alpha$ have bridging Fe atoms of the same spin whereas $\alpha x x x-x x x \beta$ have bridging Fe atoms of opposite spin. We have loosely adapted the BS numeric label syntax of each broken symmetry state, e.g., BS34 $(\alpha \alpha \beta-\alpha \beta \beta \alpha)$ from the literature on analogous $\left[\mathrm{Fe}_{4} \mathrm{~S}_{4}\right]$ clusters [32]. Our numeric labels are extended to two FeS clusters, where the first number refers to the spin states of Fe atoms 1234 and the second number refers to the spin states of Fe atoms 5678. For the low spin $(S=0)$ BS states, the following classifications can be made: (1) BS1(4-6) are states for which Fe atoms 1234 are in a $\beta \beta \alpha \alpha$ configuration; (2) BS2(4-6) are states for which Fe atoms 1234 are in a $\beta \alpha \beta \alpha$ configuration; (3) BS3(4-6) are states for which Fe atoms 1234 are in a $\beta \alpha \alpha \beta$ configuration. For the states which have Fe atoms 5678 in high spin $(S=9 / 2)$ (Figure 4 grey shaded area, right panel), the BS(78) states, the Fe atoms 1234 follow the low spin pattern of the states in the same row in Figure 4; the high spin states differ only in spin across the bridging Fe atoms, Fe1 and Fe8. Similarly, for the states which have Fe atoms 1234 in high spin $(S=9 / 2)$ (Figure 4 grey shaded area, top panel, BS4(4-6) and BS5(4-6) states), the Fe atoms 5678 follow the same spin pattern from low spin states of the states in the same column in Figure 4, differing only in spin across the bridging Fe atoms, Fe1 and Fe8.

According to Figure 1, Fe atoms 1,2,7,8 are located on the top-face and Fe atoms 3,4,5,6 are located on the bottom-face, though $\mathrm{Fe} 1$ and $\mathrm{Fe} 8$ are unique due their positions with respect to the bridging $S$ atom. For a HS A-cluster $(\alpha \alpha \alpha \alpha)$, six low-spin, BS B-cluster spin configurations were constructed; analogously, for a HS B-cluster, six BS A-cluster spin configurations were constructed.

The largest overall energy splitting is expected from the difference in energy between the high spin state $\mathrm{E}(\mathrm{HS}=\alpha \alpha \alpha \alpha-\alpha \alpha \alpha \alpha)$ and a $S=0$ broken symmetry state, e.g., $\mathrm{E}(\mathrm{BS}=\alpha \beta \alpha \beta-\alpha \beta \alpha \beta)$ (Figure 3). Within a single cubane cluster, the splitting between the HS $(\alpha \alpha \alpha \alpha)$ and BS (e.g., $\beta \alpha \beta \alpha)$ states is given by:

$$
\mathrm{E}_{\mathrm{HS}}\left(S_{\max }=9 / 2+9 / 2=18 / 2\right)-\mathrm{E}_{\mathrm{BS}}\left(\mathrm{M}_{\mathrm{BS}}=9 / 2-9 / 2=0\right)=2 \mathrm{~J}(9 / 2)(9 / 2)=(81 / 2) \mathrm{J} \text {. }
$$


The energy coupling within a single cubane, $\mathrm{J}_{\text {cubane, }}$ of DCC can be obtained through an average over all $\mathrm{E}_{\mathrm{HS}}$ corresponding to one high-spin cubane (A-cluster with $\alpha \alpha \alpha \alpha$ ) and the second cubane in all possible high and low spin BS states (Figure 3).

Within the "four-spin model", the combined spins of two Fe dimers from a single cubane cluster can couple with the spin of a dimer on the adjacent cubane cluster via the bridging sulfur atom. For a low-spin scenario, this coupling, $\mathrm{J}_{\text {cubane-cubane, is calculated }}$ from one cubane in a given low-spin BS state (e.g., A-cluster with $\beta \alpha \beta \alpha$ ), interacting with $+9 / 2$ (ferromagnetic) or $-9 / 2$ (antiferromagnetic) spin on the adjacent cubane Fe dimer via the bridging sulfur:

$$
\mathrm{E}_{\mathrm{F}}\left(\mathrm{M}_{\mathrm{BS}}=9 / 2+9 / 2=18 / 2\right)-\mathrm{E}_{\mathrm{AF}}\left(\mathrm{M}_{\mathrm{BS}}=9 / 2-9 / 2=0\right)=2 \mathrm{~J}_{\text {cubane-cubane }}(9 / 2)(9 / 2) \text {. }
$$

For a high-spin coupling scenario, coupling of the high spin cluster $(\alpha \alpha \alpha \alpha)$ with $+9 / 2$ or $-9 / 2$ spin via the bridging sulfur is given by

$$
\mathrm{E}_{\mathrm{F}}\left(\mathrm{M}_{\mathrm{BS}}=18 / 2+9 / 2=27 / 2\right)-\mathrm{E}_{\mathrm{AF}}\left(\mathrm{M}_{\mathrm{BS}}=18 / 2-9 / 2=9 / 2\right)=2 \mathrm{~J}_{\text {cubane-cubane }}(9)(9 / 2) \text {. }
$$

By considering an average of $\Delta \mathrm{E}_{\mathrm{F}-\mathrm{AF}}$, the value of $\mathrm{J}_{\text {ave }}$ may be determined [31]. The calculation of $\mathrm{J}$ from $\Delta \mathrm{E}$ follows the convention that $\mathrm{J}>0$ for antiferromagnetic (antiparallel) exchange $\mathrm{J}<0$ for ferromagnetic (parallel) exchange.

\section{Discussion}

\subsection{Exchange Parameters $J_{\text {cubane }}$ and $J_{\text {cubane-cubane }}$}

The energies of all 24 quantum mechanically (QM) optimized spin states are listed in Table S1 of the Supplementary Materials. To calculate the coupling within a single cubane cluster, $\mathrm{E}_{\mathrm{HS}}(\alpha \alpha \alpha \alpha-\mathrm{xxxx})-\mathrm{E}_{\mathrm{BS}}(\beta \alpha \beta \alpha-\mathrm{xxxx})=(81 / 2) \mathrm{J}_{\text {cubane }}, \mathrm{E}_{\mathrm{HS}}$ was first computed from an average over the 14 considered $\alpha \alpha \alpha \alpha$-xxxx states (HS1, HS2, and all grey shaded states from Figure 4) ( $E_{H S}=-16561.708$ hartree). The low spin cubane energy $E_{B S}$ was analogously computed from an average over ten possible $\beta \alpha \beta \alpha$-xxxx states (states within white area of Figure 4) $\left(\mathrm{E}_{\mathrm{BS}}=-16561.778\right.$ hartree). These values yield $\mathrm{J}_{\text {cubane }}=381 \mathrm{~cm}^{-1}$, in excellent agreement with both previous computational [23,31] as well as experimental [32-35] studies of $\left[\mathrm{Fe}_{4} \mathrm{~S}_{4}\right]^{2+}$ clusters.

To calculate the exchange parameter $\mathrm{J}_{\text {cubane-cubane, }}$ an average was constructed from the HS and BS J $\mathrm{J}_{\text {cubane-cubane }}$ parameters. For the HS states corresponding to $\Delta \mathrm{E}=81$ $\mathrm{J}_{\text {cubane-cubane }}$ (Figure 3, HS splitting), we followed a similar approach by considering the average energy of all 13 spin states corresponding to $\left(\alpha \alpha \alpha \alpha\right.$-xxxx); a value of $\mathrm{J}(\mathrm{HS})_{\text {cubane-cubane }}$ $=243 \mathrm{~cm}^{-1}$ was obtained. The BS exchange parameter $\Delta \mathrm{E}=(81 / 2) \mathrm{J}(\mathrm{BS})_{\text {cubane-cubane }}$ was computed analogously from the difference in average energy of 22 BS states $(\beta \alpha \beta \alpha$-xxxx or $x x x x-\beta \alpha \beta \alpha)\left(E_{B S}=-16561.741\right.$ hartree $)$ and ten low spin states ( $E_{B S}=-16561.778$ hartree); $\mathrm{J}(\mathrm{BS})_{\text {cubane-cubane }}=201 \mathrm{~cm}^{-1}$. Finally, an average of the HS and BS $\mathrm{J}_{\text {cubane-cubane }}$ values yields $222 \mathrm{~cm}^{-1}$, which is on the order of the calculated $\left(156 \mathrm{~cm}^{-1}\right)$ [31] and experimental $\left(100 \mathrm{~cm}^{-1}\right)[35,36]$ values for a ligand (CO)-bound 2-Fe subcluster linked to a $\left[\mathrm{Fe}_{4} \mathrm{~S}_{4}\right]$ cubane, indicating a robust treatment of the spin coupling in the eight Fe-center DCCP. Interestingly, in absence of a ligand, $\mathrm{J}_{\text {cubane-cubane }}$ for the 2-Fe subcluster linked to a $\left[\mathrm{Fe}_{4} \mathrm{~S}_{4}\right]$ cubane is found to be ten times smaller [31,35,36]. Upon ligand binding, J increases due to a change in the electronic distribution in the 2-Fe cluster. Specifically, prior to ligand binding at the distal Fe site, the unpaired electron is located at the distal Fe; following binding, the unpaired electron is located at the proximal Fe [35]. Similar arguments can be invoked to explain the magnitude of $\mathrm{J}_{\text {cubane-cubane }}$ associated with four coupled Fe-Fe dimers in DCC. Next, we turn our attention to hybrid quantum mechanical/molecular mechanical (QM/MM) geometry optimizations of DCCP.

\subsection{QM/MM Optimized Geometries}

$\mathrm{QM} / \mathrm{MM}$ optimized geometries from the nine $(S=0)$ broken symmetry states were analyzed to deduce stabilizing structural features. The nine initial spin configurations were maintained throughout the whole geometry optimization, so that each final wave function 
describes each state's geometry and spin density properties uniquely. For the lowest energy state, BS25, the corresponding spin densities after optimization are reported in Table 1 and are shown in Figure 5.

Table 1. Electronic state analysis of the spin densities of the BS25 state after geometry optimization. $\alpha$ is conventionally indicated as being positive and $\beta$ negative.

\begin{tabular}{cccccccccc}
\hline BS25 & Fe1 & Fe2 & Fe3 & Fe4 & Fe5 & Fe6 & Fe7 & Fe8 & S9 \\
\hline-2.81 & 2.93 & -2.91 & 2.99 & -3.01 & 2.87 & -2.97 & 2.90 & 0.08 \\
\hline$\beta$ & $\alpha$ & $\beta$ & $\alpha$ & $\beta$ & $\alpha$ & $\beta$ & $\alpha$ & - \\
\hline
\end{tabular}

BS25

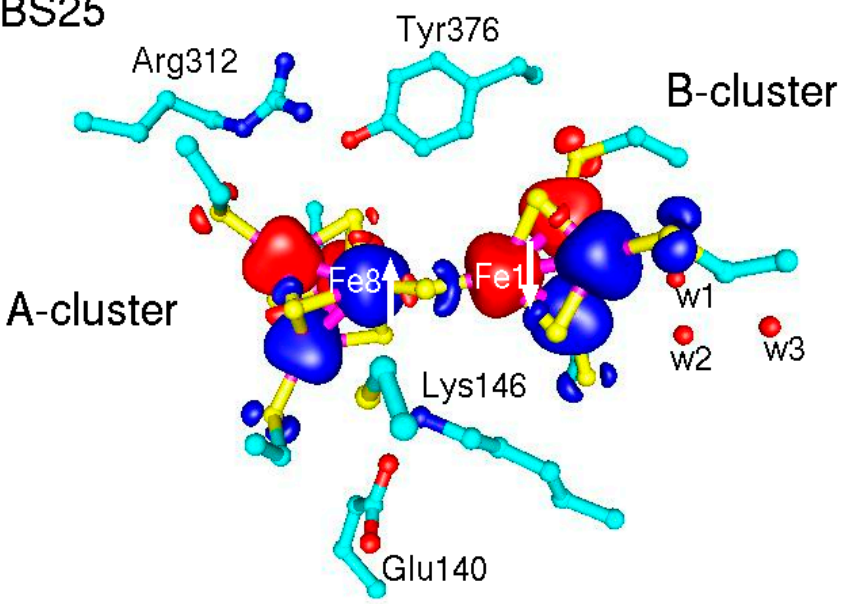

Figure 5. The BS25 spin density distribution on Fe atoms after geometry optimization. Up spin $\alpha /+$ is shown in blue, while $\beta /$ - is shown in red. Sulfur atoms are colored yellow, carbon atoms cyan, nitrogen atoms blue, and oxygen atoms in red. Hydrogen atoms are omitted for clarity. Figure drawn using gopenMol and TGIF.

The active sites of all nine $\mathrm{QM} / \mathrm{MM}$ broken symmetry states show small root mean square deviation (RMSD) (0.1 $⿱$ ) from the active site geometry in the crystal structure (PDB: 6ENO) [1]. The cubane clusters of all QM/MM optimized states preserve the starting crystal geometry (RMSD changes ranging between $0.10 \AA$ and $0.17 \AA$ ) (Table 2). In other studies of BS configurations of $\mathrm{Fe}_{4} \mathrm{~S}_{4}$ atoms, similarly minimal structural differences were observed [31]. Small structural rearrangements in surrounding protein atoms are observed in all nine states: (1) a shift $(0.7 \AA)$ of one of the oxygen atoms from a nearby water molecule (w1) to bring it closer to the peptide backbone of Cys113; (2) a twist of the sidechain of Tyr376 away from its initial position, leading to a $0.7 \AA$ movement of the hydroxyl oxygen atom to bring it closer to the positively charged sidechain of Arg312 (Arg312-Cz-OTyr376 is $3.31 \AA$ in the crystal structure and $\sim 2.89 \AA$ after QM/MM geometry optimization); (3) a small shift of Glu140 sidechain (Figure 6, shown for lowest energy state, BS25). 
Table 2. Relative quantum mechanical/molecular mechanical $(\mathrm{QM} / \mathrm{MM})$ and $\mathrm{QM}$ energy differences ( $\mathrm{kcal} / \mathrm{mol})$ and average QM/MM bond lengths $(\AA)$ for each cubane cluster are reported for each of the nine $\mathrm{BS}(S=0)$ configurations.

\begin{tabular}{|c|c|c|c|c|c|c|}
\hline BS State & $\begin{array}{l}\text { Configuation } \\
\text { Fe 1234-5678 }\end{array}$ & $\begin{array}{c}\Delta \text { Energy } \\
\text { (kcal/mol) } \\
\text { QM/MM }\end{array}$ & $\begin{array}{c}\Delta \text { Energy } \\
(\mathrm{kcal} / \mathrm{mol}) \\
\mathrm{QM}\end{array}$ & $\begin{array}{c}\text { Average } \\
\text { Cluster A } \\
\text { Bond Length } \\
\text { (̊̊) }\end{array}$ & $\begin{array}{c}\text { Average } \\
\text { Cluster B Bond } \\
\text { Length }(\AA ̊)\end{array}$ & $\begin{array}{c}\text { Average } \\
\text { Cluster Bond } \\
\text { Length (̊̊) }\end{array}$ \\
\hline BS25 & $\beta \alpha \beta \alpha-\beta \alpha \beta \alpha$ & 0.00 & 2.037 & 2.269 & 2.263 & 2.266 \\
\hline BS15 & $\beta \beta \alpha \alpha-\beta \alpha \beta \alpha$ & 0.45 & 2.517 & 2.271 & 2.269 & 2.270 \\
\hline BS35 & $\beta \alpha \alpha \beta-\beta \alpha \beta \alpha$ & 1.34 & 2.443 & 2.267 & 2.270 & 2.268 \\
\hline BS24 & $\beta \alpha \beta \alpha-\alpha \beta \beta \alpha$ & 2.30 & 0.517 & 2.269 & 2.262 & 2.265 \\
\hline BS34 & $\beta \alpha \alpha \beta-\alpha \beta \beta \alpha$ & 2.37 & 1.347 & 2.269 & 2.262 & 2.265 \\
\hline BS36 & $\beta \alpha \alpha \beta-\beta \beta \alpha \alpha$ & 2.38 & 0.979 & 2.266 & 2.263 & 2.265 \\
\hline BS26 & $\beta \alpha \beta \alpha-\beta \beta \alpha \alpha$ & 2.59 & 0.556 & 2.266 & 2.263 & 2.265 \\
\hline BS14 & $\beta \beta \alpha \alpha-\alpha \beta \beta \alpha$ & 2.95 & 0.812 & 2.270 & 2.269 & 2.269 \\
\hline BS16 & $\beta \beta \alpha \alpha-\beta \beta \alpha \alpha$ & 4.27 & 0.00 & 2.267 & 2.270 & 2.268 \\
\hline
\end{tabular}

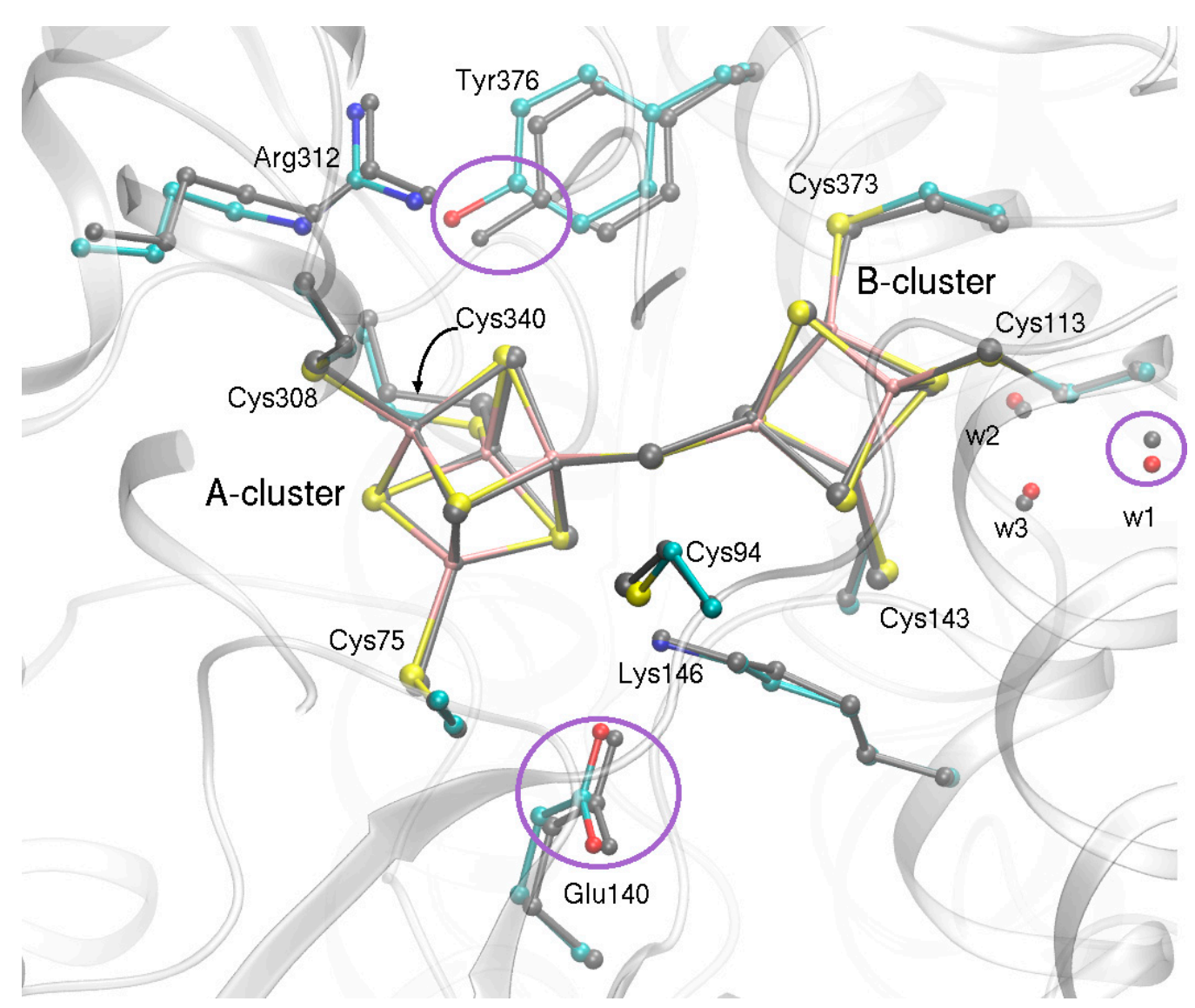

Figure 6. For BS25, the QM/MM geometry optimized active site (in color) is compared to the crystal structure geometry (PDB: 6ENO) [1] (in grey). In the QM/MM geometry optimized structure, iron atoms are colored pink, sulfur atoms are colored yellow, carbon atoms cyan, nitrogen atoms blue, and oxygen atoms in red. Hydrogen atoms are omitted for clarity. Purple circles are drawn around larger structural changes. Figure drawn using VMD and TGIF.

The relative energies of each of the nine BS states indicate that the arrangement of unpaired electrons on the Fe atoms gives rise to energy differences $(<5 \mathrm{kcal} / \mathrm{mol})$ that are 
related to the electron configuration, in agreement with $\mathrm{QM} / \mathrm{MM}$ calculations reported previously for H-cluster in Fe-only hydrogenase [37].

In each BS state, the electrons belonging to Fe ions bridging the $\mu_{2}-\mathrm{S}$ atom are spinpaired (i.e., Fe1 $\beta$ and $\mathrm{Fe} 8 \alpha$ ). In general, each symmetry state gives rise to a mixture of four short (type $\mathrm{s}=\mathrm{d}<2.25 \AA$ ) and four long (type $\mathrm{l}=\mathrm{d}>2.25 \AA$ ) Fe-S bonds (pattern shown for BS25 A-cluster in Table S2). Aside from BS35, the average bond lengths within the A-cluster are larger than those of the B-cluster (Figure S2). The average cluster bond length ranges from $2.265 \AA$ to $2.270 \AA$ (Figure S2).

Lower energy geometries can be linked to the spin densities' distributions on the different sub-clusters. The three lowest energy states, BS25 $(\alpha \beta \alpha-\beta \alpha \beta \alpha), \operatorname{BS} 15(\beta \beta \alpha \alpha-\beta \alpha \beta \alpha)$, and BS35 $(\beta \alpha \alpha \beta-\beta \alpha \beta \alpha)$ have in common the $\beta \alpha \beta \alpha$ pattern of electron spins on Fe atoms $5,6,7,8$ on the A-cluster, nearest to the residues Arg312, Glu140, Lys146 with charge-bearing side chains. These three lowest energy states demonstrate a nearly identical pattern of bond lengths on the A-cluster (Figure 7A), suggesting that this FeS cluster is highly sensitive to the charged protein environment and is the driving factor for overall DCC stability inside the protein scaffold.

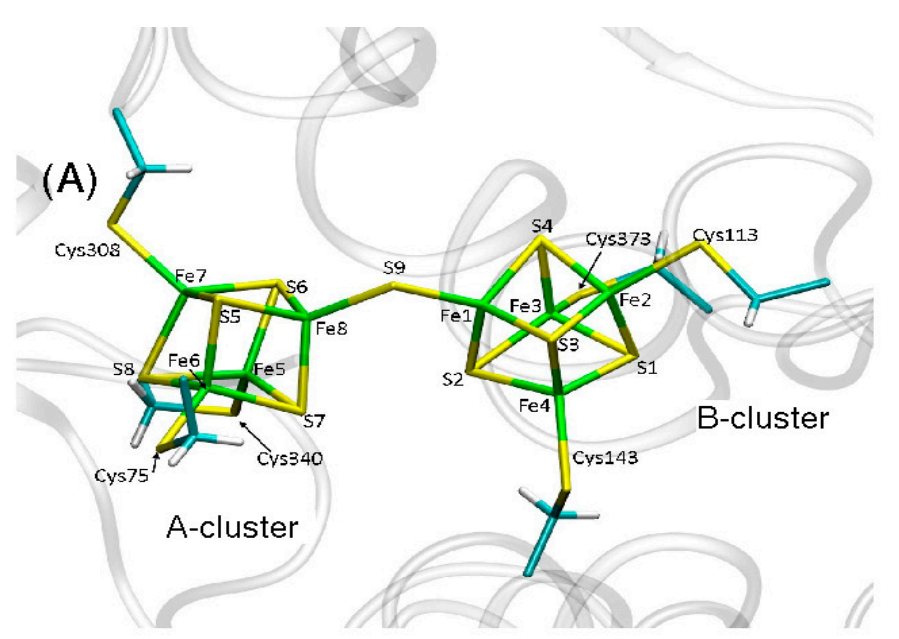

(B) in protein environment (QM/MM)

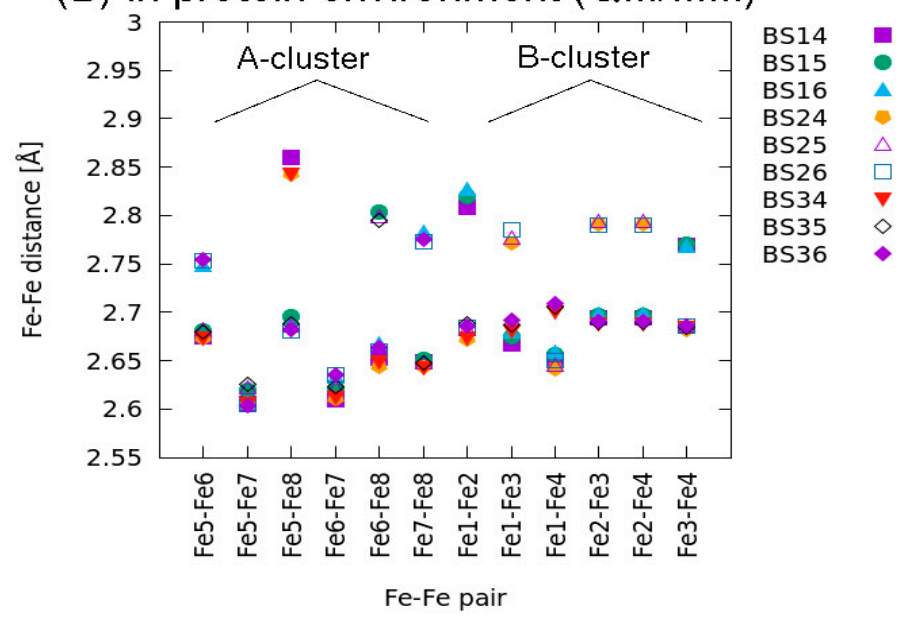

Figure 7. Cont. 


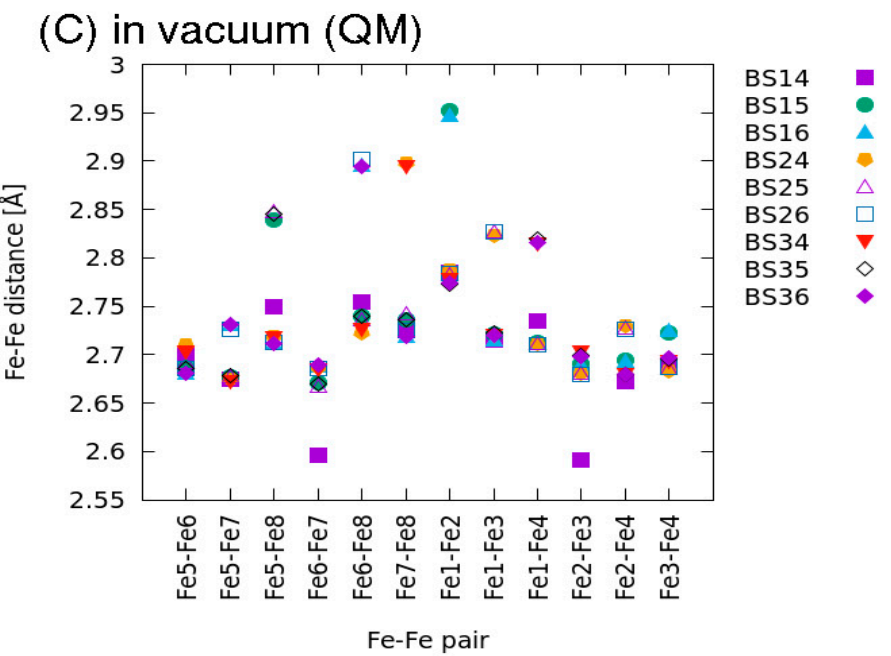

Figure 7. The distance between Fe atoms $(\AA)$ in the DCC $(\mathbf{A})$ is compared for protein environment (B) and vacuum (C). Fe-Fe distances span a larger range in the absence of the protein scaffold. Figure drawn using gnuplot and TGIF.

In general, if two electrons of the same spin are located on iron atoms on the face of the cubane cluster, a lengthening of the sides of the face is observed. This phenomenon is further enhanced in the presence of an additional electrostatic environment, e.g., if amino acids with charge-bearing sidechains are nearby. Compared to the other BS states, the three lowest energy states, BS25, BS15, BS35, demonstrate a lengthened $\mathrm{Fe} 6(\alpha)-\mathrm{Fe} 8(\alpha)$ distance $(2.80 \AA)$ and a shortened Fe5( $\beta)-\mathrm{Fe} 7(\beta)$ distance $(2.62 \AA)$ (Figure $8 \mathrm{~B})$. In other words, the iron atoms on the A-cluster face that is nearest to the charges localized on the Glu140 and Lys146 sidechains ( -1 and +1 , respectively) are farther apart than the iron atoms on the face toward the single charged sidechain of Arg312 (+1). The longest Fe-Fe separation $(\sim 2.85 \AA)$ is observed for Fe5-Fe8 of states BS14, BS24, BS34, which have in common the $(\alpha \beta \beta \alpha)$ configuration on A-cluster iron atoms Fe5, Fe6, Fe7, Fe8. Iron atoms Fe5 and Fe8 carrying $\alpha$-spin are located on the face pointing toward the B-cluster. In the absence of the protein environment, the Fe-Fe separations are largest (2.95 $⿱$ A) for BS15 and BS16 (Figure $7 \mathrm{C}$ ), whose Fe1-Fe2 atoms both carry $\beta$ spin and are located vis-à-vis the A-cluster face containing $\mathrm{Fe} 5$ and $\mathrm{Fe} 8$, which, in these two BS states, also carry an unpaired electron of $\beta$ spin. The iron atoms react to this high spin environment by seeking greater separation.
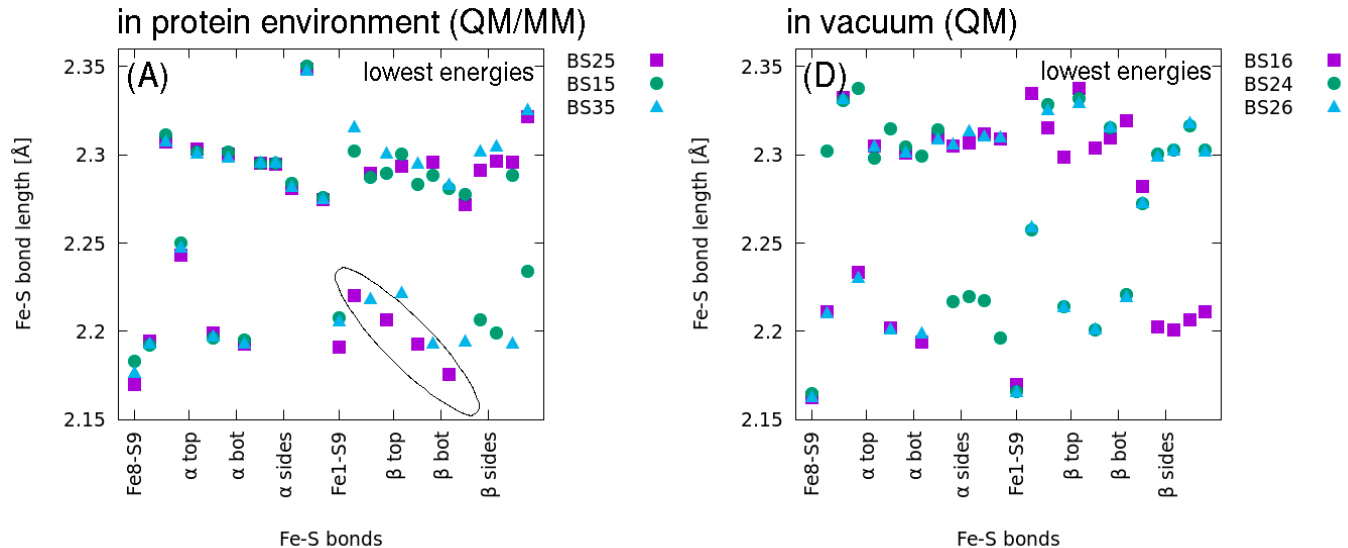

Figure 8. Cont. 

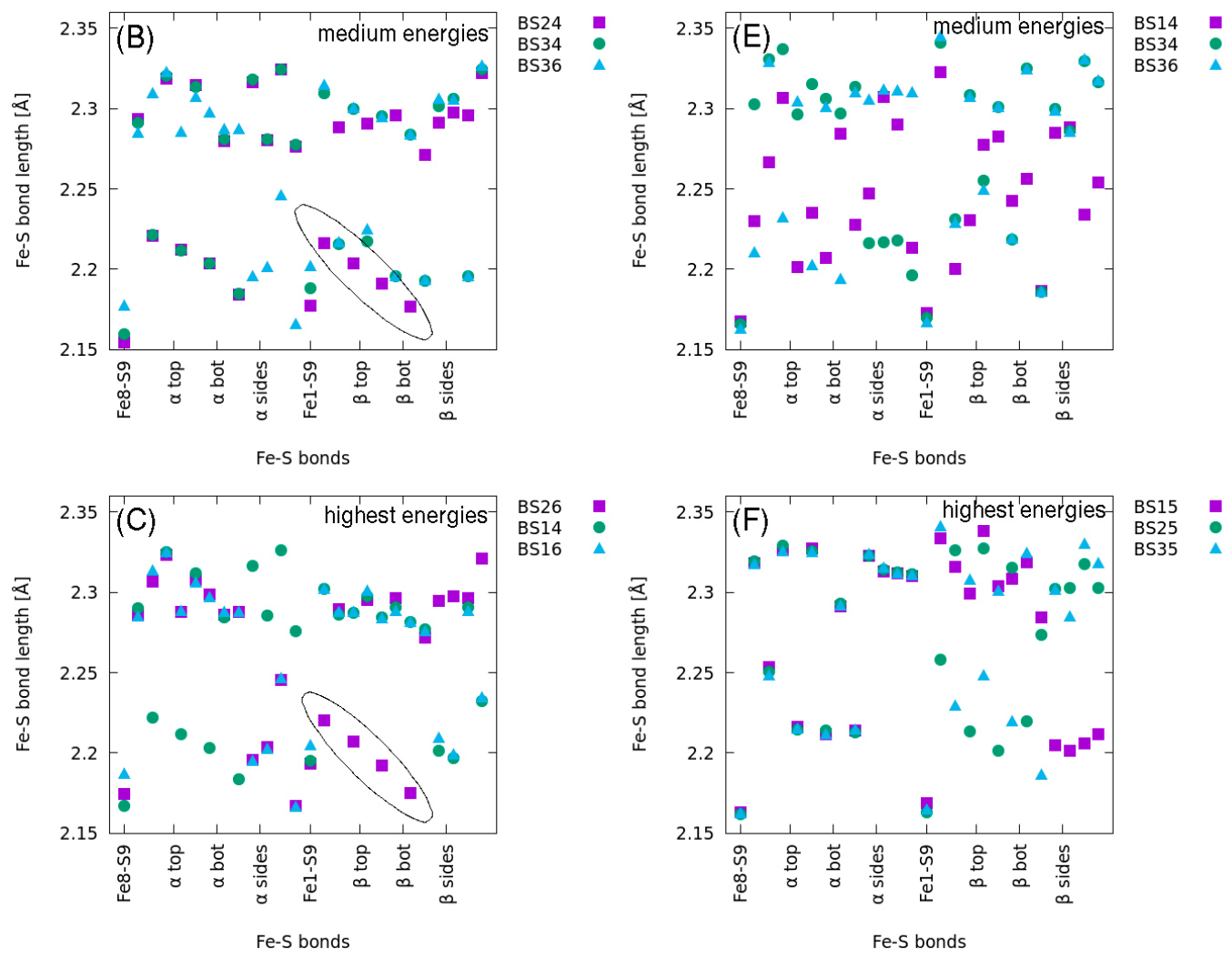

Figure 8. Fe-S bond lengths $(\AA)$ of low, medium, and highest energy BS states are compared for the DCC within (A-C) protein environment and (D-F) vacuum. The bond types are classified according to position within each cubane, "top" face, "bottom" face, "sides". In general, the electrostatic protein environment induces more systematic Fe-S bonding patterns, such as for the BS24, BS25, BS26 states (circled) in the B-cluster. Figure drawn using gnuplot and TGIF.

Compared to vacuum (Figure 8D-F), the protein environment reduces the scattering in $\mathrm{Fe}-\mathrm{S}$ bond lengths in the $\mathrm{DCC}$, and more systematic geometrical patterns emerge (Figure 8A-C). Particularly for the BS24, BS25, BS26 states, Fe-S bond lengths are conserved in the B-cluster (circled purple squares in Figure $8 \mathrm{~A}-\mathrm{C}$ ), reflecting the $\beta \alpha \beta \alpha$-spin pattern that is common to these three states (Figure 4). Interestingly, the Fe8-S9 (A-cluster Fe8 with bridging $\mu_{2}-\mathrm{S}$ atom) is consistently between $2.15-2.18 \AA$ in both protein and vacuum environments. The Fe1-S9 bond, on the other hand, is lengthened considerably in the protein scaffold, reaching values as high as $2.2 \AA$ (Figure $8 \mathrm{~A}-\mathrm{C}$ ).

Taken together, these geometry analyses indicate that electrons of the same spin in a charged environment seek maximum separation. For the BS states investigated with the DCC system, these observations explain the overall more compact nature of the B-cluster, which is not in the direct vicinity of amino acids with charge-bearing sidechains. In general, cluster reduction by the addition of an electron would increase S-S atom repulsion and lead to overall cluster expansion [38]. For the DCC system, the energetic cost of reduction may therefore be smaller if an electron is added at the B-cluster as this cluster is not surrounded by amino acids carrying charged sidechains.

\section{Methods}

\subsection{Broken Symmetry States}

In addition to the HS1 state ( $\alpha \alpha \alpha \alpha-\alpha \alpha \alpha \alpha)$, following the BS-DFT approach to multicenter transition metal clusters, various parallel (ferromagnetic) or antiparallel (antiferromagnetic) alignments of the Fe iron site spins were considered, resulting in a total number of 22 different BS states defined following the numbering of the eight Fe atoms in Figure 1, i.e., 1234-5678 (Figure 4). The semi-high spin HS2 $(\alpha \alpha \alpha \alpha-\beta \beta \beta \beta)$ was also considered (see Introduction for a detailed description). In Figure 4, the grey shaded 
area includes BS states in which one cluster is high spin and the other cluster is low spin, either $\alpha x x x-x x x \alpha$ or $\alpha x x x-x x x \beta$ across bridging $\mu_{2}-S$. The state BS52 (Figure 4) was examined only in the context of QM spin analyses. It is important to point out that, in the asymmetric protein matrix, each of these spin states is unique due to surrounding amino acids. In vacuum, six of the BS states $(44,45,46,54,55,56)$ are expected to collapse into the six of corresponding patterns $(37,27,17,38,28,18$, respectively), due to the symmetry of the DCC.

In each calculation, each of the sub-clusters is considered to be in the $\left[\mathrm{Fe}_{4} \mathrm{~S}_{4}\right]^{2+}$ oxidized state. Hence, the formal number of ferrous $\left(\mathrm{Fe}^{2+}, \mathrm{d} 6, S=2\right)$ and ferric $\left(\mathrm{Fe}^{3+}, \mathrm{d} 5, S=5 / 2\right)$ iron sites is two and two, respectively, for a total $\mathrm{M}_{\mathrm{s}}=1$ (Figure 2). For a single cubane cluster in an $\left[\mathrm{Fe}_{4} \mathrm{~S}_{4}\right]^{2+}$ oxidized state with two each ferrous $\left(\mathrm{Fe}^{2+}, \mathrm{d} 6, \mathrm{~S}=2\right)$ and ferric $\left(\mathrm{Fe}^{3+}\right.$, d5 $S=5 / 2$ ) iron sites, two electron exchange phenomena should be considered [21-23]: (1) double exchange leads to ferromagnetic coupling of spins for a pair of Fe centers to yield two Fe-Fe dimers with $S=9 / 2$ each; (2) according to Heisenberg exchange, the two $S=9 / 2$ dimer spins are coupled antiferromagnetically to produce $S=0$ ground state for a total $\mathrm{M}_{\mathrm{S}}=1$ (Figure 2). Only the nine $S=0$ BS states are considered in the discussion of DCC structural features (Figure 4, black text without gray shading).

\subsection{Model for QM Geometry Optimization}

Geometry optimizations of each of the spin states were carried out on an active site containing 65 atoms in vacuum. For these calculations the models considered only the atoms comprising the DCC, including four iron and four sulfur atoms for each sub-cluster, one $\mu_{2}-\mathrm{S}$ bridging the two cubane clusters and three cysteine thiolates binding each subsystem (Cys340, Cys308, Cys75 in the A-cluster; Cys113, Cys143, Cys373 in the B-cluster). The $C-C$ covalent bonds connecting the $C_{\alpha}$ and the backbone of the six coordinating cysteine sidechains were cut and the valency of $C_{\alpha}$ atoms was satisfied by adding a proton to each of them. During geometry optimization of the DCC in vacuum, the $C_{\alpha}$ atoms were kept fixed to maintain their positions in the protein. A figure showing the 65 atoms included in the QM geometry optimization is included in the Supplementary Materials (Figure S1). The geometry optimizations, carried out for each of the nine different spin configurations, were performed using the DFT functional BP86 [39,40], def2-TZVP basis set for Fe and $S$ and 6-31g* for non-metals, respectively, combined with the resolution-of-identity (RI) technique as implemented in TURBOMOLE [41,42].

\subsection{Model for $Q M / M M$ Geometry Optimization}

In a second set of calculations, a hybrid QM/MM approach was used to investigate the effect of protein matrix on the DCC active site. For the QM/MM geometry optimizations, the system is divided into three computational layers (Figure 9). The first layer, treated at the QM level, consists of the 157 DCC atoms coordinated to subsystems (Cys340, Cys308, Cys75 in sub-cluster 1; Cys373, Cys113, Cys143 in sub-cluster 2) and atoms of sidechains (up to and including $\mathrm{C}_{\alpha}$ ) of the following amino acids: Cys94, Glu140, Lys146, Arg312, and Tyr376 (Figure 9A). The side chains of Lys146 and Arg312 were considered to carry a positive charge, while Glu140 was modeled with a negative charge. Three water molecules (w1, w2, w3) were also included in this QM region. All atoms in the first computational layer were treated with DFT using the functional BP86 [39,40], def2-TZVP basis set for Fe and $\mathrm{S}$ and $6-31 \mathrm{~g}^{*}$ for non-metals, respectively, combined with the resolution-of -identity (RI) technique as implemented in TURBOMOLE [41,42]. 
(A)

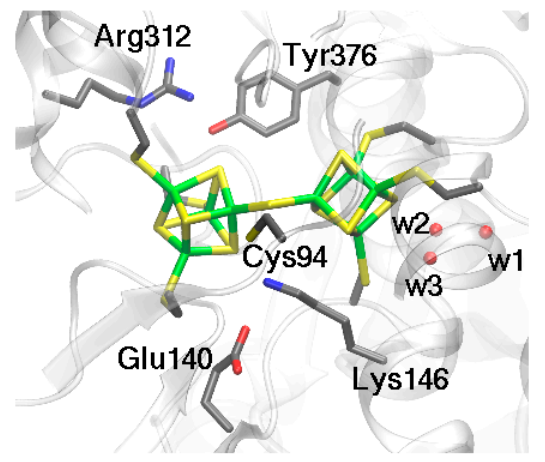

(B)
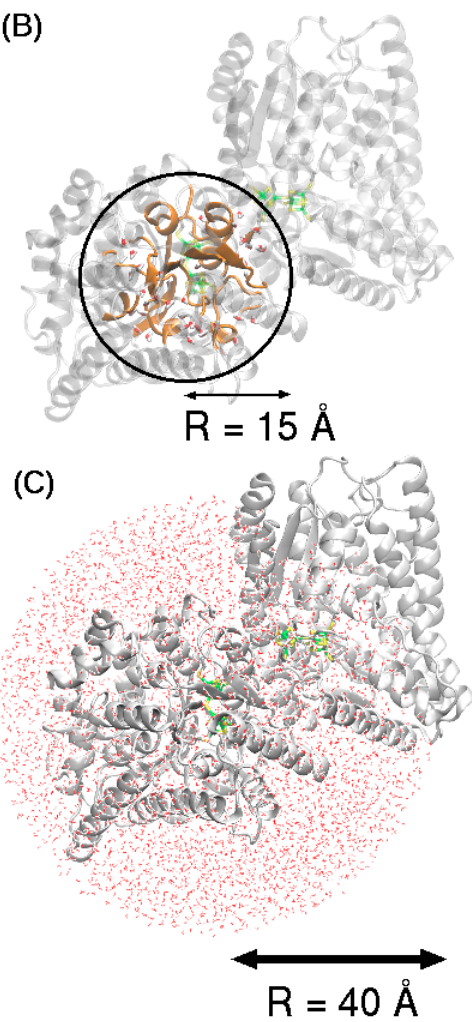

Figure 9. The $\mathrm{QM} / \mathrm{MM}$ setup of the system consists of a three-tiered approach. (A) The model of the double-cubane cluster of $\mathrm{DCCP}_{\mathrm{Ch}}$ was constructed from the structure file 6ENO.pdb (1.63 $\AA$ ) [1], coordinated to Cys75, Cys113, Cys143, Cys308, Cys340 and Cys373. A-cluster is linked to B-cluster via the $\mu_{2}$-S ligand (S9). (B) The second layer of the model includes all protein and water atoms within a radius of $15 \AA$ from the iron atom Fe8 located on the A-cluster. (C) The third layer includes all remaining protein, solvent, and salt ions within a $40 \AA$ radius of Fe8; these atoms were held fixed during all geometry optimizations. Sulphur atoms are colored yellow; iron atoms are green; carbon atoms are cyan, and hydrogen atoms are white.

The second layer of the model includes all protein and water atoms within a radius of $15 \AA$ from the iron atom Fe8 located on the A-cluster. All atoms within this second layer were treated explicitly using the charmm22/CMAP force field [43] and allowed to relax during the geometry optimization cycles. The third layer includes all remaining protein, solvent, and salt ions extending $40 \AA$ from Fe8 (Figure 9C); the atoms in this third layer were held fixed during the optimization procedure.

The protein model for DCC was constructed using the $1.63 \AA$ structure (6ENO.pdb) obtained through X-ray diffraction [1]. All enzyme atoms, including hydrogen atoms, were modeled with CHARMM [43], using the charmm22/CMAP force field [43,44]. Point charges of the DCC cluster atoms with coordinating Cys residues were computed from the electrostatic potential (ESP), as implemented in Gaussian16 [45] using DFT with the functional BP86 [40], def2-TZVP basis set for Fe and S and 6-31g* for non-metals. The van der Waals parameters for the Fe atoms were assigned values reported in the literature for Fe in similar enzymatic systems [46].

The enzyme was next solvated in a box of dimension $105 \AA \times 119 \AA \times 121 \AA$ with 42,393 explicit TIP3 water molecules [47], 32 sodium ions, and eight chlorine ions to neutralize charge. The positions of protein hydrogen atoms and solvent water atoms were optimized using a conjugate gradient energy minimization routine, as implemented in NAMD [48].

The charmm22/CMAP force field was used to describe all bonding and non-bonding interactions. Covalent bonds traversing the QM-MM border (between the $C_{\alpha}$ and $C_{\beta}$ ) were cut and hydrogen link-atoms were inserted to satisfy valency. According to established 
$\mathrm{QM} / \mathrm{MM}$ protocols, the energetic coupling between the first and second layers (QM and mobile $\mathrm{MM}$ ) was treated using electrostatic embedding with a charge-shift scheme as implemented in ChemShell $[49,50]$. For each of the nine BS states considered here, a geometry optimization was carried out.

\section{Conclusions}

Using a single-determinant DFT approach, the ground electronic state of spin-coupled systems cannot be described, as the state is defined by a combination of multiple determinants. Within the framework of the BS formalism, however, these spin interactions can be modeled by localizing opposite spins of the wave function in different parts of the molecule. For enzymes with multi-metal centers, such as the novel multicenter transition metal cluster DCC, this approach provides us access to the lowest energy state as well as the exchange parameters between coupled spins. Here, 23 spin states were studied, and the calculated $\mathrm{J}_{\text {cubane }}$ and $\mathrm{J}_{\text {cubane-cubane }}$ show excellent agreement with values determined computationally and experimentally for analogous FeS systems. QM and QM/MM geometry optimizations were carried out for nine BS states. The protein scaffold was shown to play an essential role in determining the lowest energy state.

The catalytic activity of $\mathrm{DCCP}_{\mathrm{Ch}}$ protein containing the DCC is comparable to the activity of enzymes such as the nitrogenases, which are able to reduce small molecules such as acetylene. In addition to this similar catalytic activity, the geometric symmetry of the P-cluster of nitrogenase suggests that the $8 \mathrm{Fe}$ cluster is formed from two closely-spaced $4 \mathrm{Fe}$ clusters [51]. Moreover, the precursor to the P-cluster is catalytically active and catalyzes the reduction of $\mathrm{CO}$ and $\mathrm{CN}$ to alkanes and alkenes [52]. These striking analogies suggest that DCCP and nitrogenase may have common ancestors. Here, the focus of the investigation was to provide the community with (1) an initial characterization of the DCC spin states, and (2) QM/MM optimized geometries of the DCCP active site based on a BS wavefunction. With this foundation, ongoing investigations are aimed at modeling substrate and inhibitor molecules, including acetylene and CO. Also, the protein scaffold's effect on the reduction potential in DCC will be characterized. DCCP may therefore serve as a model for a class of DCC-containing enzymes with unexplored reactivity and enzymatic potential.

Supplementary Materials: The following are available online at https: / www.mdpi.com/2073-4 344/11/2/245/s1, Figure S1: DCC atoms considered in QM region, Figure S2: QM/MM geometry optimized average cluster bond lengths; Table S1: QM energies. Table S2: Fe-S bond lengths of A-subcluster of BS25.

Author Contributions: Investigation, N.E.-M., D.T., M.A.M.; writing-original draft preparation, N.E.-M., D.T.; writing—review and editing, N.E.-M., D.T., M.A.M.; funding acquisition, M.A.M. All authors have read and agreed to the published version of the manuscript.

Funding: This research was funded by the Deutsche Forschungsgemeinschaft (DFG, German Research Foundation) under Germany's Excellence Strategy-EXC 2008-390540038-UniSysCat.

Data Availability Statement: The data presented in this study are available on request from the corresponding author.

Acknowledgments: The authors thank I. Zebger and C. Kulka at the Technische Universität Berlin for insightful discussions.

Conflicts of Interest: The authors declare no conflict of interest.

\section{References}

1. Jeoung, J.-H.; Dobbek, H. ATP-dependent substrate reduction at an [Fe8S9] double-cubane cluster. Proc. Natl. Acad. Sci. USA 2018, 115, 2994-2999. [CrossRef]

2. Jeoung, J.; Martins, B.M.; Dobbek, H. Double-Cubane [8Fe9S] Clusters: A Novel Nitrogenase-Related Cofactor in Biology. ChemBioChem 2020, 21, 1710-1716. [CrossRef] [PubMed]

3. Stack, T.D.; Carney, M.J.; Holm, R.H. Formation of Bridged [4Fe-4S]2+ Double Cubanes by Site-Specific Reactions: Elec-tronTransfer Coupling Across Sulfur Containing Bridges of Variable Length. J. Am. Chem. Soc. 1989, 111, 1670-1676. [CrossRef] 
4. Terada, T.; Wakimoto, T.; Nakamura, T.; Hirabayashi, K.; Tanaka, K.; Li, J.; Matsumoto, T.; Tatsumi, K.; Matsumoto, T.; Tatsumi, K. Tridentate Thiolate Ligands: Application to the Synthesis of the Site-Differentiated [4Fe-4S] Cluster having a Hydrosulfide Ligand at the Unique Iron Center. Chem. Asian J. 2012, 7, 920-929. [CrossRef] [PubMed]

5. Gerlach, D.L.; Coucouvanis, D.; Kampf, J.; Lehnert, N. Isolation and Characterization of Single and Sulfide-Bridged Double [4Fe-4S] Cubane Clusters with 4-Pyridinethiolato Ligands. Eur. J. Inorg. Chem. 2013, 2013, 5253-5264. [CrossRef]

6. Challen, P.R.; Koo, S.-M.; Dunham, R.; Coucouvanis, D. New $\mu 2-S 2$-Coupled, Singly Bridged Double Cubane with the [(Fe4S4Cl3)2S]4- Core. The Stepwise Synthesis and Structural Characterization of (n -Bu4N)2 (Ph4P)2[(Fe4S4C13)2S]. J. Am. Chem. Soc. 1990, 112, 2455-2456. [CrossRef]

7. Lee, S.C.; Lo, W.; Holm, R.H. Developments in the Biomimetic Chemistry of Cubane-Type and Higher Nuclearity Iron-Sulfur Clusters. Chem. Rev. 2014, 114, 3579-3600. [CrossRef]

8. Rao, P.V.; Holm, R.H. Synthetic Analogues of the Active Sites of Iron-Sulfur Proteins. Chem. Rev. 2004, 104, 527-560. [CrossRef]

9. Somasegaran, P.; Hoben, H. Handbook for Rhizobia: Methods in Legume-rhizobium Technology; Springer Lab Manuals Series; Springer: Berlin/Heidelberg, Germany, 1994.

10. Lee, H.-I.; Sørlie, M.; Christiansen, J.; Song, R.; Dean, D.R.; Hales, B.J.; Hoffman, B.M. Characterization of an Intermediate in the Reduction of Acetylene by the Nitrogenase $\alpha$-Gln195MoFe Protein by Q-band EPR and13C,1H ENDOR. J. Am. Chem. Soc. 2000, 122, 5582-5587. [CrossRef]

11. Seefeldt, L.C.; Hoffman, B.; Dean, D.R. Mechanism of Mo-Dependent Nitrogenase. Annu. Rev. Biochem. 2009, 78, 701-722. [CrossRef]

12. Gao, H.; Azam, T.; Randeniya, S.; Couturier, J.; Rouhier, N.; Johnson, M.K. Function and maturation of the Fe-S center in dihydroxyacid dehydratase fromArabidopsis. J. Biol. Chem. 2018, 293, 4422-4433. [CrossRef]

13. Katz, S.; Noth, J.; Horch, M.; Shafaat, H.; Happe, T.; Hildebrandt, P.; Zebger, I. Vibrational spectroscopy reveals the initial steps of biological hydrogen evolution. Chem. Sci. 2016, 7, 6746-6752. [CrossRef] [PubMed]

14. Lubitz, W.; Reijerse, E.J.; Van Gastel, M. [NiFe] and [FeFe] Hydrogenases Studied by Advanced Magnetic Resonance Techniques. Chem. Rev. 2007, 107, 4331-4365. [CrossRef]

15. DeLacey, A.L.; Fernández, V.M.; Rousset, M.; Cammack, R. Activation and inactivation of hydrogenase function and the catalytic cycle: Spectrochemical studies. Chem. Rev. 2007, 107, 4304-4330. [CrossRef]

16. Spiro, T.G.; Czernuszewicz, R.S. Biochemical Spectroscopy; Methods in Enzymology; Academic Press: Cambridge, MA, USA, 1995; Volume 246, pp. 416-460.

17. Todorovic, S.; Teixeira, M. Resonance Raman spectroscopy of Fe-S proteins and their redox properties. J. Biol. Inorg. Chem. 2018, 23, 647-661. [CrossRef]

18. Glaser, T. Mössbauer Spectroscopy and Transition Metal Chemistry. Fundamentals and Applications; Gütlich, P., Bill, E., Trautwein, A.X., Eds.; Springer: Berlin/Heidelberg, Germany, 2011.

19. Frielingsdorf, S.; Fritsch, J.; Schmidt, A.; Hammer, M.; Löwenstein, J.; Siebert, E.; Pelmenschikov, V.; Jaenicke, T.; Kalms, J.; Rippers, Y.; et al. Reversible [4Fe-3S] cluster morphing in an O2-tolerant [NiFe] hydrogenase. Nat. Chem. Biol. 2014, 10, 378-385. [CrossRef] [PubMed]

20. Roessler, M.M.; Salvadori, E. Principles and applications of EPR spectroscopy in the chemical sciences. Chem. Soc. Rev. 2018, 47, 2534-2553. [CrossRef]

21. Noodleman, L. Valence bond description of antiferromagnetic coupling in transition metal dimers. J. Chem. Phys. 1981, 74, 5737-5743. [CrossRef]

22. Noodleman, L.; Baerends, E.J. Electronic structure, magnetic properties, ESR, and optical spectra for 2-iron ferredoxin models by LCAO-X $\alpha$ valence bond theory. J. Am. Chem. Soc. 1984, 106, 2316-2327. [CrossRef]

23. Noodleman, L.; Peng, C.; Case, D.; Mouesca, J.-M. Orbital interactions, electron delocalization and spin coupling in iron-sulfur clusters. Co-ord. Chem. Rev. 1995, 144, 199-244. [CrossRef]

24. Roos, B.O.; Taylor, P.R.; Sigbahn, P.E.M. A complete active space SCF method (CASSCF) using a density matrix formulated super-CI approach. Chem. Phys. 1980, 48, 157-173. [CrossRef]

25. Sharma, S.; Sivalingam, K.; Neese, F.; Chan, G.K.-L. Low-energy spectrum of iron-sulfur clusters directly from many-particle quantum mechanics. Nat. Chem. 2014, 6, 927-933. [CrossRef] [PubMed]

26. Cho, D.; Rouxel, J.R.; Mukamel, S.; Chan, G.K.-L.; Li, Z. Stimulated X-ray Raman and Absorption Spectroscopy of Iron-Sulfur Dimers. J. Phys. Chem. Lett. 2019, 10, 6664-6671. [CrossRef]

27. Li, Z.; Guo, S.; Sun, Q.; Chan, G.K.-L. Electronic landscape of the P-cluster of nitrogenase as revealed through many-electron quantum wavefunction simulations. Nat. Chem. 2019, 11, 1026-1033. [CrossRef]

28. Kubas, A. Characterization of charge transfer excited states in [2Fe-2S] iron-sulfur clusters using conventional configuration interaction techniques. Theor. Chem. Accounts 2020, 139, 1-7. [CrossRef]

29. Papaefthymiou, V.; Millar, M.M.; Münck, E. Mössbauer and EPR studies of a synthetic analogue for the Fe4S4 core of oxidized and reduced high-potential iron proteins. Inorg. Chem. 1986, 25, 3010-3014. [CrossRef]

30. Cammack, R. Iron-Sulfur Clusters in Enzymes: Themes and Variations. Adv. Inorg. Chem. 1992, 38, 281-322. [CrossRef]

31. Fiedler, A.T.; Brunold, T.C. Computational Studies of the H-Cluster of Fe-Only Hydrogenases: Geometric, Electronic, and Magnetic Properties and Their Dependence on the [Fe4S4] Cubane. Inorg. Chem. 2005, 44, 9322-9334. [CrossRef] 
32. Volbeda, A.; Amara, P.; Darnault, C.; Mouesca, J.-M.; Parkin, A.; Roessler, M.M.; Armstrong, F.A.; Fontecilla-Camps, J.C. X-ray crystallographic and computational studies of the O2-tolerant [NiFe]-hydrogenase 1 from Escherichia coli. Proc. Nat. Aca. Sci. USA 2012, 109, 5305-5310. [CrossRef]

33. Papaefthymiou, G.C.; Laskowski, E.J.; Frota-Pessôa, S.; Frankel, R.B.; Holm, R.H. Antiferromagnetic exchange interactions in [Fe4S4(SR)4]2-,3- clusters. Inorg. Chem. 1982, 21, 1723-1728. [CrossRef]

34. Laskowski, E.J.; Frankel, R.B.; Gillum, W.O.; Papaefthymiou, G.C.; Renaud, J.; Ibers, J.A.; Holm, R.H. Synthetic analogs of the 4-Fe active sites of reduced ferredoxins. Electronic properties of the tetranuclear trianions [Fe4S4(SR)4]3- and the structure of [(C2H5)3(CH3)N]3[Fe4S4(SC6H5)4]. J. Am. Chem. Soc. 1978, 100, 5322-5337. [CrossRef]

35. Pereira, A.S.; Tavares, P.; Moura, I.; Moura, J.J.; Huynh, B.H. Mössbauer characterization of the iron-sulfur clusters in Desulfovibrio vulgaris hydrogenase. J. Am. Chem. Soc. 2001, 123, 2771-2782. [CrossRef] [PubMed]

36. Popescu, C.V.; Münck, E. Electronic Structure of the H Cluster in [Fe]-Hydrogenases. J. Am. Chem. Soc. 1999, 121, 7877-7884. [CrossRef]

37. Greco, C.; Bruschi, M.; De Gioia, L.; Ryde, U. A QM/MM Investigation of the Activation and Catalytic Mechanism of Fe-Only Hydrogenases. Inorg. Chem. 2007, 46, 5911-5921. [CrossRef]

38. Mouesca, J.-M.; Chen, J.L.; Noodleman, L.; Bashford, N.; Case, D.A. Density Functional/Poisson-Boltzmann Calculations of Redox Potentials for Iron-Sulfur Clusters. J. Am. Chem. Soc. 1994, 116, 11898-11914. [CrossRef]

39. Becke, A.D. Density-functional exchange-energy approximation with correct asymptotic behavior. Phys. Rev. A 1988, 38, 3098-3100. [CrossRef]

40. Perdew, J.P. Density-functional approximation for the correlation energy of the inhomogeneous electron gas. Phys. Rev. B 1986, 33, 8822-8824. [CrossRef]

41. Ahlrichs, R.; Bär, M.; Häser, M.; Horn, C.K.H. Electronic structure calculations on work-station computers: The computer system turbomole. Chem. Phys. Lett. 1989, 162, 165-169. [CrossRef]

42. TURBOMOLE V6.2 2010, A Development of University of Karlsruhe and Forschungszentrum Karlsruhe GmbH, 1989-2007, TURBOMOLE GmbH, Since 2007. 2010. Available online: http:/ / www.turbomole.com (accessed on 9 February 2021).

43. MacKerell, A.D., Jr.; Feig, M.; Brooks, C.L., III. Extending the treatment of backbone energetics in protein force fields: Limitations of gas-phase quantum mechanics in reproducing protein conformational distributions in molecular dynamics simulations. J. Comput. Chem. 2004, 25, 1400-1415. [CrossRef]

44. Brooks, B.R.; Brooks, C.L., III; MacKerell, A.D.; Nilsson, L.; Petrella, R.J.; Roux, B.; Won, Y.; Archontis, G.; Bartels, C.; Boresch, S.; et al. CHARMM: The Biomolecular simulation Program. J. Comp. Chem. 2009, 30, 1545-1615. [CrossRef]

45. Frisch, M.J.; Trucks, G.W.; Schlegel, H.B.; Scuseria, G.E.; Robb, M.A.; Cheeseman, J.R.; Scalmani, G.; Barone, V.; Petersson, G.A.; Nakatsuji, H.; et al. Gaussian16 Revision; Gaussian, Inc.: Wallingford, CT, USA, 2016.

46. Rappé, A.K.; Casewit, C.J.; Colwell, K.S.; Goddard, W.A., III. UFF, a full periodic table force field for molecular mechanics and molecular dynamics simulations. J. Am. Chem. Soc. 1992, 114, 10024-10035. [CrossRef]

47. Jorgensen, W.L.; Chandrasekhar, J.; Madura, J.D.; Impey, R.W.; Klein, M.L. Comparison of simple potential functions for simulating liquid water. J. Chem. Phys. 1983, 79, 926-935. [CrossRef]

48. Phillips, J.C.; Hardy, D.J.; Maia, J.D.C.; Stone, J.E.; Ribeiro, J.V.; Bernardi, R.C.; Buch, R.; Fiorin, G.; Hénin, J.; Jiang, W.; et al. Scalable molecular dynamics on CPU and GPU architectures with NAMD. J. Chem. Phys. 2020, 153, 044130. [CrossRef] [PubMed]

49. Sherwood, P.; DeVries, A.H.; Guest, M.F.; Schreckenbach, G.; Catlow, C.R.A.; French, S.A.; Sokol, A.A.; Bromley, S.T.; Thiel, W.; Turner, A.J.; et al. QUASI: A general purpose implementation of the QM/MM approach and its application to problems in catalysis. J. Mol. Struct. 2003, 632, 1-28. [CrossRef]

50. Metz, S.; Kästner, A.; Sokol, A.; Keal, T.W.; Sherwood, P. ChemShell-A modular software package for QM/MM simulations, Wiley Interdisc. Rev. Comp. Mol. Sci. 2014, 4, 101-110. [CrossRef]

51. Ribbe, M.W.; Hu, Y.; Hodgson, K.O.; Hedman, B. Biosynthesis of Nitrogenase Metalloclusters. Chem. Rev. 2014, 114, 4063-4080. [CrossRef] [PubMed]

52. Lee, C.C.L.; Hu, Y.; Ribbe, M.W. ATP-independent substrate reduction by nitrogenase P-cluster variant. Proc. Nat. Aca. Sci. USA 2012, 109, 6922-6926. [CrossRef] [PubMed] 